\title{
FILTER SPACES AND BASICALLY DISCONNECTED COVERS
}

\author{
Young Ju JEON ${ }^{\mathrm{a}}$ AND ChANGIL Kim ${ }^{\mathrm{b}, *}$
}

\begin{abstract}
In this paper, we first show that for any space $X$, there is a $\sigma$-complete Boolean subalgebra $Z\left(\Lambda_{X}\right)^{\#}$ of $\mathcal{R}(X)$ and that the subspace $\{\alpha \mid \alpha$ is a fixed $\sigma Z(X)^{\#}$-ultrafilter $\}$ of the Stone-space $S\left(Z\left(\Lambda_{X}\right)^{\#}\right)$ is the minimal basically disconnected cover of $X$. Using this, we will show that for any countably locally weakly Lindelöf space $X$, the set $\{M \mid M$ is a $\sigma$-complete Boolean subalgebra of $\mathcal{R}(X)$ containing $Z(X)^{\#}$ and $s_{M}^{-1}(X)$ is basically disconnected $\}$, when partially ordered by inclusion, becomes a complete lattice.
\end{abstract}

\section{INTRODUCTION}

All spaces in this paper are Tychonoff spaces and $\beta X$ denotes the Stone-Čech compactification of a space $X$.

Vermeer $([10])$ showed that every space $X$ has the minimal basically disconnected cover $\left(\Lambda X, \Lambda_{X}\right)$ and if $X$ is a compact space, then $\Lambda X$ is given by the Stone-space $S\left(\sigma Z(X)^{\#}\right)$ of a $\sigma$-complete Boolean subalgebra $\sigma Z(X)^{\#}$ of $\mathcal{R}(X)$. Henriksen, Vermeer and Woods([4])(Kim [7], resp.) showed that the

minimal basically disconnected cover of a weakly Lindelöf space (a locally weakly Lindelöf space, resp.) $X$ is given by the subspace $\left\{\alpha \mid \alpha\right.$ is a fixed $\sigma Z(X)^{\#}$ ultrafilter $\}$ of the Stone-space $S\left(\sigma Z(X)^{\#}\right)$.

In this paper, we first show that for any space $X$, there is a $\sigma$-complete Boolean subalgebra $Z\left(\Lambda_{X}\right)^{\#}$ of $\mathcal{R}(X)$ and that the subspace $\left\{\alpha \mid \alpha\right.$ is a fixed $\sigma Z(X)^{\#}$. ultrafilter $\}$ of the Stone-space $S\left(Z\left(\Lambda_{X}\right)^{\#}\right)$ is the the minimal basically disconnected cover of $X$. Using this, we will show that $S\left(Z\left(\Lambda_{X}\right)^{\#}\right)$ and $\beta \Lambda X$ are homeomorphic. Moreover, we show that for any $\sigma$-complete Booeal subalgebra $M$ of $\mathcal{R}(X)$ containing $Z(X)^{\#}$, the Stone-space $S(M)$ of $M$ is a basically diconnected cover of $X$ and that the subspace $\{\alpha \mid \alpha$ is a fixed $M$-ultrafilter $\}$ of the Stone-space $S(M)$ is the the

Received by the editors January 10, 2014. Revised March 4, 2014. Accepted March 10, 2014. 2010 Mathematics Subject Classification. 54G05, 54G99, 54C01.

Key words and phrases. basically disconnected cover, Stone-space, covering map.

${ }^{*}$ Corresponding author. 
minimal basically disconnected cover of $X$ if and only if it is a basically disconnected space and $M \subseteq Z\left(\Lambda_{X}\right)^{\#}$. Finally, we will show that for any countably locally weakly Lindelöf space $X$, the set $\{M \mid M$ is a $\sigma$-complete Boolean subalgebra of $\mathcal{R}(X)$ containg $Z(X)^{\#}$ and $s_{M}^{-1}(X)$ is basically disconnected\}, when partially ordered by inclusion, becomes a complete lattice.

For the terminology, we refer to [1] and [9].

\section{Filter Spaces}

The set $\mathcal{R}(X)$ of all regular closed sets in a space $X$, when partially ordered by inclusion, becomes a complete Boolean algebra, in which the join, meet, and complementation operations are defined as follows : for any $A \in \mathcal{R}(X)$ and any $\left\{A_{i}: i \in I\right\} \subseteq \mathcal{R}(X)$,

$$
\begin{aligned}
& \vee\left\{A_{i}: i \in I\right\}=c l_{X}\left(\cup\left\{A_{i}: i \in I\right\}\right), \\
& \wedge\left\{A_{i}: i \in I\right\}=c l_{X}\left(i n t_{X}\left(\cap\left\{A_{i}: i \in I\right\}\right)\right), \text { and } \\
& A^{\prime}=c l_{X}(X-A)
\end{aligned}
$$

and a sublattice of $\mathcal{R}(X)$ is a subset of $\mathcal{R}(X)$ that contains $\emptyset, X$ and is closed under finite joins and meets.

We recall that a map $f: Y \longrightarrow X$ is called a covering map if it is a continuous, onto, perfect, and irreducible map.

Lemma $2.1([5])$.

(1) Let $f: Y \longrightarrow X$ be a covering map. Then the map $\psi: \mathcal{R}(Y) \longrightarrow \mathcal{R}(X)$, defined by $\psi(A)=f(A) \cap X$, is a Boolean isomorphism and the inverse map $\psi^{-1}$ of $\psi$ is given by $\psi^{-1}(B)=c l_{Y}\left(f^{-1}\left(\right.\right.$ int $\left.\left._{X}(B)\right)\right)=c l_{Y}\left(\right.$ int $\left._{Y}\left(f^{-1}(B)\right)\right)$.

(2) Let $X$ be a dense subspace of a space $K$. Then the map $\phi: \mathcal{R}(K) \longrightarrow \mathcal{R}(X)$, defined by $\phi(A)=A \cap X$, is a Boolean isomorphism and the inverse map $\phi^{-1}$ of $\phi$ is given by $\phi^{-1}(B)=c_{K}(B)$.

A lattice $L$ is called $\sigma$-complete if every countable subset of $L$ has the join and the meet. For any subset $M$ of a Boolean algebra $L$, there is the smallest $\sigma$-complete Boolean subalgebra $\sigma M$ of $L$ containing $M$. Let $X$ be a space and $Z(X)$ the set of all zero-sets in $X$. Then $Z(X)^{\#}=\left\{c l_{X}\left(\right.\right.$ int $\left.\left._{X}(Z)\right) \mid Z \in Z(X)\right\}$ is a sublattice of $\mathcal{R}(X)$.

We recall that a subspace $X$ of a space $Y$ is $C^{*}$-embedded in $Y$ if for any realvalued continuous map $f: X \longrightarrow \mathbb{R}$, there is a continuous map $g: Y \longrightarrow \mathbb{R}$ such that $\left.g\right|_{X}=f$. 
Let $X$ be a space. Since $X$ is $C^{*}$-embedded in $\beta X$, by Lemma 2.1., $\sigma Z(X)^{\#}$ and $\sigma Z(\beta X)^{\#}$ are Boolean isomorphic.

Let $X$ be a space and $\mathcal{B}$ a Boolean subalgebra of $\mathcal{R}(X)$. Let $S(\mathcal{B})=\{\alpha \mid \alpha$ is a $\mathcal{B}$-ultrafilter $\}$ and for any $B \in \mathcal{B}, \Sigma_{B}^{\mathcal{B}}=\{\alpha \in S(\mathcal{B}) \mid B \in \alpha\}$. Then the space $S(\mathcal{B})$, equipped with the topology for which $\left\{\Sigma_{B}^{\mathcal{B}} \mid B \in \mathcal{B}\right\}$ is a base, called the Stone-space of $\mathcal{B}$. Then $S(\mathcal{B})$ is a compact, zero-dimensional space and the map $s_{\mathcal{B}}: S(\mathcal{B}) \longrightarrow \beta X$, defined by $s_{\mathcal{B}}(\alpha)=\cap\left\{c l_{\beta X}(A) \mid A \in \mathcal{B}\right\}$, is a covering map ([7]).

Definition 2.2. A space $X$ is called basically disconnected if for any zero-set $Z$ in $X, \operatorname{int}_{X}(Z)$ is closed in $X$, equivalently, every cozero-set in $X$ is $C^{*}$-embedded in $X$.

A space $X$ is a basically disconnected space if and only if $\beta X$ is a basically disconnected space. If $X$ is a basically disconnected space, every element in $Z(X)^{\#}$ is clopen in $X$ and so $X$ is a basically disconnected space if and only if $Z(X)^{\#}$ is a $\sigma$-complete Boolean algebra.

Definition 2.3. Let $X$ be a space. Then a pair $(Y, f)$ is called

(1) a cover of $X$ if $f: Y \longrightarrow X$ is a covering map,

(2) a basically disconnected cover of $X$ if $(Y, f)$ is a cover of $X$ and $Y$ is a basically disconnected space, and

(3) a minimal basically disconnected cover of $X$ if $(Y, f)$ is a basically disconnected cover of $X$ and for any basically disconnected cover $(Z, g)$ of $X$, there is a covering map $h: Z \longrightarrow Y$ such that $f \circ h=g$.

Vermeer $([10])$ showed that every space $X$ has a minimal basically disconnected cover $\left(\Lambda X, \Lambda_{X}\right)$ and that if $X$ is a compact space, then $\Lambda X$ is the Stone-space $S\left(\sigma Z(X)^{\#}\right)$ of $\sigma Z(X)^{\#}$ and $\Lambda_{X}(\alpha)=\cap\{A \mid A \in \alpha\}(\alpha \in \Lambda X)$.

Let $X$ be a space. Since $\sigma Z(X)^{\#}$ and $\sigma Z(\beta X)^{\#}$ are Boolean isomorphic, $S\left(\sigma Z(X)^{\#}\right)$ and $S\left(\sigma Z(\beta X)^{\#}\right)$ are homeomorphic.

Let $X, Y$ be spaces and $f: Y \longrightarrow X$ a map. For any $U \subseteq X$, let $f_{U}: f^{-1}(U) \longrightarrow$ $U$ denote the restriction and co-restriction of $f$ with respect to $f^{-1}(U)$ and $U$, respectively.

In the following, for any space $X,\left(\Lambda \beta X, \Lambda_{\beta}\right)$ denotes the minimal basically disconnected cover of $\beta X$.

Lemma $2.4([7])$. Let $X$ be a space. If $\Lambda_{\beta}^{-1}(X)$ is a basically disconnected space, then $\left(\Lambda_{\beta}^{-1}(X), \Lambda_{\beta_{X}}\right)$ is the minimal basically disconnected cover of $X$. 
For any covering map $f: Y \longrightarrow X$, let $Z(f)^{\#}=\left\{c l_{Y}\left(\right.\right.$ int $\left.\left._{X}(f(Z))\right) \mid Z \in Z(Y)^{\#}\right\}$. Since $\mathcal{R}(\Lambda X)$ and $\mathcal{R}(X)$ are Boolean isomorphic and $Z(\Lambda X)^{\#}$ is a $\sigma$-complete Boolean subalgebra of $\mathcal{R}(\Lambda X)$, by Lemma $2.1, Z\left(\Lambda_{X}\right)^{\#}$ is a $\sigma$-complete Boolean subalgebra of $\mathcal{R}(X)$.

Definition 2.5. Let $X$ be a space and $\mathcal{B}$ a sublattice of $\mathcal{R}(X)$. Then a $\mathcal{B}$-filter $\mathcal{F}$ is called fixed if $\{F \mid F \in \mathcal{F}\} \neq \emptyset$.

Let $X$ be a space and for any $Z\left(\Lambda_{X}\right)^{\#}$-ultrafilter $\alpha$, let $\alpha_{\lambda}=\left\{A \in Z(\Lambda X)^{\#} \mid\right.$ $\left.\Lambda_{X}(A) \in \alpha\right\}$.

Proposition 2.6. Let $X$ be a space and $\alpha$ a fixed $Z\left(\Lambda_{X}\right)^{\#}$-ultrafilter. Then $\alpha_{\lambda}$ is a fixed $Z(\Lambda X)^{\#}$-ultrafilter and $\left|\cap\left\{A \mid A \in \alpha_{\lambda}\right\}\right|=1$.

Proof. Clearly, $\alpha_{\lambda}$ is a $Z(\Lambda X)^{\#}$-filter. Suppose that $A \in Z(\Lambda X)^{\#}-\alpha_{\lambda}$. Then $\Lambda_{X}(A) \in Z\left(\Lambda_{X}\right)^{\#}-\alpha$. Since $\alpha$ is a $Z\left(\Lambda_{X}\right)^{\#}$-ultrafilter, there is a $C \in \alpha$ such that $C \wedge \Lambda_{X}(A)=\emptyset$ and hence $A \wedge c l_{\Lambda X}\left(\Lambda_{X}^{-1}\left(\operatorname{int}_{X}(C)\right)\right)=\emptyset$. Since $\Lambda_{X}\left(\operatorname{cl}_{\Lambda X}\left(\Lambda_{X}^{-1}\left(\right.\right.\right.$ int $\left.\left.\left._{X}(C)\right)\right)\right)=$ $C \in \alpha, c l_{\Lambda X}\left(\Lambda_{X}^{-1}\left(\right.\right.$ int $\left.\left._{X}(C)\right)\right) \in \alpha_{\lambda}$ and hence $\alpha_{\lambda}$ is a $Z(\Lambda X)^{\#}$-ultrafilter. Since $\alpha$ is fixed, there is an $x \in \cap\{B \mid B \in \alpha\}$. Then $\left\{A \cap \Lambda_{X}^{-1}(x) \mid A \in \alpha_{\lambda}\right\}$ has a family of closed sets in $\Lambda_{X}^{-1}(x)$ with the finite intersection property. Since $\Lambda_{X}^{-1}(x)$ is a compact subset of $\Lambda X, \cap\left\{A \cap \Lambda_{X}^{-1}(x) \mid A \in \alpha_{\lambda}\right\} \neq \emptyset$ and hence $\cap\left\{A \mid A \in \alpha_{\lambda}\right\} \neq \emptyset$. Since $Z(\Lambda X)^{\#}$ is a base for $\Lambda X$ and $\alpha_{\lambda}$ is a $Z(\Lambda X)^{\#}$-ultrafilter, $\left|\cap\left\{A \mid A \in \alpha_{\lambda}\right\}\right|=1$.

Let $X$ be a space and $F X=\left\{\alpha \mid \alpha\right.$ is a fixed $Z\left(\Lambda_{X}\right)^{\#}$-ultrafilter $\}$ the subspace of the Stone space $S\left(Z\left(\Lambda_{X}\right)^{\#}\right)$. Define a map $h_{X}: F X \longrightarrow \Lambda X$ by $h_{X}(\alpha)=\cap\{A \mid$ $\left.A \in \alpha_{\lambda}\right\}$. In the following, let $\Sigma_{B}=\Sigma_{B}^{Z\left(\Lambda_{X}\right)^{\#}}$ for any $B \in Z\left(\Lambda_{X}\right)^{\#}$.

Theorem 2.7. Let $X$ be a space. Then $h_{X}: F X \longrightarrow \Lambda X$ is a homeomorphism.

Proof. Take any $\alpha, \delta$ in $F X$ with $\alpha \neq \delta$. Since $\alpha$ and $\delta$ are $Z\left(\Lambda_{X}\right)^{\#}$-ultrafilters, there are $A, B$ in $Z(\Lambda X)^{\#}$ such that $\Lambda_{X}(A) \in \alpha, \Lambda_{X}(B) \in \delta$ such that $\Lambda_{X}(A) \wedge \Lambda_{X}(B)=\emptyset$. Then $A \in \alpha_{\lambda}, B \in \delta_{\lambda}$ and $A \wedge B=\emptyset$. By Lemma 2.1, $c l_{\Lambda X}(A) \cap c l_{\Lambda X}(B)=\emptyset$ and $h_{X}(\alpha)=\cap\left\{G \mid G \in \alpha_{\lambda}\right\} \neq \cap\left\{H \mid H \in \delta_{\lambda}\right\}=h_{X}(\delta)$. Thus $h_{X}$ is an one-to-one map.

Let $y \in \Lambda X$ and $\gamma=\left\{\Lambda_{X}(C) \mid y \in C \in Z(\Lambda X)^{\#}\right\}$. Since every element of $Z(\Lambda X)^{\#}$ is a clopen set in $\Lambda X, \gamma \in F X$ and $h_{X}(\gamma)=y$ and hence $h_{X}$ is an onto map.

Let $E \in Z(\Lambda X)^{\#}$. Suppose that $\mu \in F X-h_{X}^{-1}(E)$. Since $\Lambda_{X}(E) \notin \mu, \mu \notin \Sigma_{\Lambda_{X}(E)}$ and so $\Sigma_{\Lambda_{X}}(E) \subseteq h^{-1}(E)$. Suppose that $\theta \in h_{X}^{-1}(E)$. Then $h_{X}(\theta) \in E$ and hence for any $A \in \theta_{\lambda}, A \cap E \neq \emptyset$. Since $\theta_{\lambda}$ is a $Z(\Lambda X)^{\#}$-ultrafilter, $E \in \theta_{\lambda}$ and so $E \in \Sigma_{\Lambda X(E)}$ 
and $h_{X}(\theta) \in E$. Hence $\Sigma_{\Lambda_{X}(E)}=h_{X}^{-1}(E)$ and since $h_{X}$ is one-to-one and onto, $h_{X}$ is a homeomorphism.

Corollary 2.8. Let $X$ be a space and $F_{X}=\Lambda_{X} \circ h_{X}$. Then $\left(F X, F_{X}\right)$ is the minimal basically disconnected cover of $X$ and $F(\alpha)=\cap\{A \mid A \in \alpha\}$ for all $\alpha \in F X$.

It is well-known that a space $X$ is $C^{*}$-embedded in its compactification $Y$ if and only if $\beta X=Y$.

Theorem 2.9. Let $X$ be a space. Then there is a homeomorphism $k: \beta \Lambda X \longrightarrow$ $S\left(Z\left(\Lambda_{X}\right)^{\#}\right)$ such that $k \circ \beta_{\Lambda X} \circ h_{X}=j$, where $j: F X \longrightarrow S\left(Z\left(\Lambda_{X}\right)^{\#}\right)$ is the inclusion map.

Proof. By Theorem 2.7., $\beta F X=\beta \Lambda X$ and $S\left(Z\left(\Lambda_{X}\right)^{\#}\right)$ is a compactification of $F X$. Hence there is a continuous map $k: \beta \Lambda X \longrightarrow S\left(Z\left(\Lambda_{X}\right)^{\#}\right)$ such that $k \circ \beta_{\Lambda X} \circ h_{X}=j$, where $j: \Lambda X \longrightarrow S\left(Z\left(\Lambda_{X}\right)^{\#}\right)$ is the dense embedding. Let $T=S\left(Z\left(\Lambda_{X}\right)^{\#}\right)$ and $A, B$ be disjoint zero-sets in $F X$. Then there are disjoint zero-sets $C, D$ in $F X$ such that $A \subseteq \operatorname{int}_{F X}(C)$ and $B \subseteq \operatorname{int}_{F X}(D)$. Since $h_{X}: F X \longrightarrow \Lambda X$ is a homeomorphism, $c l_{F X}\left(\operatorname{int}_{F X}(C)\right)=\Sigma_{F_{X}\left(c l_{F X}\left(\text { int }_{F X}(C)\right)\right)} \cap F X$ and since $F X$ is dense in $T, \operatorname{cl}_{T}\left(\operatorname{cl}_{F X}\left(\operatorname{int}_{F X}(C)\right)\right)=\Sigma_{F_{X}\left(c l_{F X}\left(\operatorname{int}_{F X}(C)\right)\right)}$. Similarly,

$$
c l_{T}\left(c l_{F X}\left(\operatorname{int}_{F X}(D)\right)\right)=\Sigma_{F_{X}\left(c_{F X}\left(\operatorname{int}_{F X}(D)\right)\right)} .
$$

Since $\left.\left.c l_{F X}\left(\operatorname{int}_{F X}(C)\right)\right) \wedge c l_{F X}\left(\operatorname{int}_{F X}(D)\right)\right)=\emptyset$,

$$
F_{X}\left(\operatorname{cl}_{F X}\left(\operatorname{int}_{F X}(C)\right)\right) \wedge F_{X}\left(c l_{F X}\left(\operatorname{int}_{F X}(D)\right)\right)=\emptyset .
$$

Hence

$$
\begin{aligned}
& c l_{T}\left(\operatorname{cl}_{F X}\left(\operatorname{int}_{F X}(C)\right)\right) \cap c l_{T}\left(\operatorname{cl}_{F X}\left(\operatorname{int}_{F X}(D)\right)\right) \\
& =\Sigma_{F_{X}\left(c l_{F X}\left(\operatorname{int}_{F X}(C)\right)\right)} \cap \Sigma_{F_{X}\left(c l_{F X}\left(\text { int }_{F X}(D)\right)\right)} \\
& =\Sigma_{F_{X}\left(c l_{\Lambda X}\left(\operatorname{int}_{\Lambda X}(C)\right)\right) \wedge F_{X}\left(c l_{F X}\left(\operatorname{int}_{F X}(D)\right)\right)} \\
& =\emptyset \text {. }
\end{aligned}
$$

By the Uryshon's extension theorem, $F X$ is $C^{*}$-embedded in $T$ and so $k$ is a homeomorphism.

It is known that $\beta \Lambda X=\Lambda \beta X$ if and only if $\left\{\Lambda_{X}(A) \mid A \in Z(\Lambda X)^{\#}\right\}=$ $\sigma Z(X)^{\#}([5])$. Hence we have the following :

Corollary 2.10. Let $X$ be a space. Then $\beta \Lambda X=\Lambda \beta X$ if and only if $Z\left(\Lambda_{X}\right)^{\#}=$ $\sigma Z(X)^{\# \text {. }}$ 


\section{Basically Disconnected Covers}

Let $X$ be a space and $M$ a $\sigma$-complete Boolean subalgebra of $\mathcal{R}(X)$ containg $Z(X)^{\#}$. By the dfinition of $\sigma Z(X)^{\#}, \sigma Z(X)^{\#} \subseteq M$.

Proposition 3.1. Let $X$ be a space and $M$ a $\sigma$-complete Boolean subalgebra of $\mathcal{R}(X)$ containg $Z(X)^{\#}$. Then $S(M)$ is a basically disconnected space.

Proof. Let $D$ be a cozero-set in $S(M)$. Since $S(M)$ is a compact space, $D$ is a Lindelöf space and hence there is a sequense $\left(A_{n}\right)$ in $M$ such that $D=\cup\left\{\Sigma_{A_{n}}^{M} \mid n \in\right.$ $N\}$. Clearly, $c l_{S(M)}(D) \subseteq \Sigma_{\vee\left\{A_{n} \mid n \in N\right\}}^{M}$. Let $\alpha \in S(M)-c l_{S(M)}\left(\cup\left\{\Sigma_{A_{n}}^{M} \mid n \in N\right\}\right)$. Then there is a $B \in M$ such that $\alpha \in \Sigma_{B}^{M}$ and $\left(\cup\left\{\Sigma_{A_{n}}^{M} \mid n \in N\right\}\right) \cap \Sigma_{B}^{M}=\emptyset$. Hence for any $n \in N, \Sigma_{A_{n}}^{M} \cap \Sigma_{B}^{M}=\Sigma_{A_{n} \wedge B}=\emptyset$ and hence $A_{n} \wedge B=\emptyset$. So, $\vee\left\{A_{n} \wedge B \mid n \in N\right\}=\left(\vee\left\{A_{n} \mid n \in N\right\}\right) \wedge B=\emptyset$. Since $B \in \alpha, \vee\left\{A_{n} \mid n \in N\right\} \notin \alpha$ and so $\alpha \notin \Sigma_{\vee\left\{A_{n} \mid n \in N\right\}}$. Hence $c_{S(M)}(D)$ is open in $S(M)$ and thus $S(M)$ is a basically disconnected space.

Let $X$ be a space and $M$ a $\sigma$-complete Boolean subalgebra of $\mathcal{R}(X)$ containg $Z(X)^{\#}$. By Theorem 3.1, there is a covering map $t: S(M) \longrightarrow \Lambda \beta X$ such that $\Lambda_{\beta} \circ t=s_{M}$.

Theorem 3.2. Let $X$ be a space and $M$ a $\sigma$-complete Boolean subalgebra of $\mathcal{R}(X)$ containg $Z(X)^{\#}$. Then we have the following :

(1) There is a covering map $g: S(M) \longrightarrow \beta \Lambda X$ such that $s_{Z\left(\Lambda_{X}\right)}{ }^{\#} \circ g=s_{M}$ if and only if $Z\left(\Lambda_{X}\right)^{\#} \subseteq M$.

(2) There is a covering map $f: \beta \Lambda X \longrightarrow S(M)$ such that $s_{M} \circ f=s_{Z\left(\Lambda_{X}\right)} \#$ if and only if $M \subseteq Z\left(\Lambda_{X}\right)^{\#}$.

(3) $\left(s_{M}^{-1}(X), s_{M_{X}}\right)$ is the minimal basically disconnected cover of $X$ if and only if $\left(s_{M}^{-1}(X), s_{M_{X}}\right)$ is a basically disconnected cover of $X$ and $M \subseteq Z\left(\Lambda_{X}\right)^{\#}$.

Proof. (1) $(\Rightarrow)$ Take any $Z \in Z(\Lambda X)^{\#}$. Then there is an $A \in Z(\beta \Lambda X)^{\#}$ such that $Z=A \cap \Lambda X$. Since $\beta \Lambda X$ is basically disconnected, $g^{-1}(A)$ is a clopen-set in $S(M)$. Since $S(M)$ is compact, there is a $D \in M$ such that $g^{-1}(A)=\Sigma_{D}^{M}$. Since $s_{M}$ and $s_{Z\left(\Lambda_{X}\right)^{\#}}$ are covering maps, $c l_{\beta X}(D)=s_{M}\left(g^{-1}(A)\right)=s_{Z\left(\Lambda_{X}\right) \#}(A)$. By Lemma 2.1, $D=s_{M}\left(g^{-1}(A)\right) \cap X=s_{Z\left(\Lambda_{X}\right)}(A) \cap X=\Lambda_{X}(A \cap \Lambda X)=\Lambda_{X}(Z)$ and hence $\Lambda_{X}(Z) \in M$.

$(\Leftarrow)$ It is trivial $([9])$.

Similarly, we have (2) 
(3) $(\Rightarrow)$ Suppose that $\left(s_{M}^{-1}(X), s_{M_{X}}\right)$ is the minimal basically disconnected cover of $X$. Then there is a homeomorhpism $l: s_{M}^{-1}(X) \longrightarrow \Lambda X$ such that $\Lambda_{X} \circ l=s_{M_{X}}$. Hence there is a covering map $f: \beta \Lambda X \longrightarrow S(M)$ such that $f \circ \beta_{\Lambda X} \circ l=j$, where $j: s_{M}^{-1}(X) \longrightarrow S(M)$ is the inclusion map. Take any $D \in M$. Then $f^{-1}\left(\Sigma_{D}^{M}\right)$ is a clopen set in $\beta \Lambda X$ and since $\beta \Lambda X$ is a compact space, there is an $A \in Z\left(\Lambda_{X}\right)^{\#}$ such that $\Sigma_{A}=f^{-1}\left(\Sigma_{D}^{M}\right)$. Hence $s_{Z\left(\Lambda_{X}\right) \#}\left(\Sigma_{A}\right)=c l_{\beta X}(A)=s_{Z\left(\Lambda_{X}\right) \#}\left(f^{-1}\left(\Sigma_{D}^{M}\right)\right)$. Since $s_{M} \circ f \circ \beta_{\Lambda X} \circ l=s_{M} \circ j=\beta_{X} \circ \Lambda_{X} \circ l=s_{Z\left(\Lambda_{X}\right) \#} \circ \beta_{\Lambda X} \circ l$ and $\beta_{\Lambda X} \circ l$ is a dense embedding, $s_{M} \circ f=s_{Z\left(\Lambda_{X}\right)}$. By $(2)$, we have the result.

$(\Leftarrow)$ Since $s_{M}^{-1}(X)$ is a basically disconnected space, there is a covering map $l: s_{M}^{-1}(X) \longrightarrow \Lambda X$ such that $\Lambda_{X} \circ l=s_{M_{X}}$. Since $M \subseteq Z\left(\Lambda_{X}\right)^{\#}$, by (2), there is a

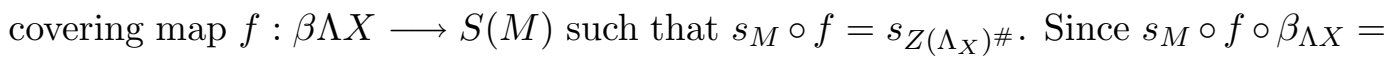
$s_{Z\left(\Lambda_{X}\right)} \circ \beta_{\Lambda X}=\beta_{X} \circ \Lambda_{X}$, there is a covering $m: \Lambda X \longrightarrow s_{M}^{-1}(X)$ such that $s_{M_{X}} \circ m=$ $\Lambda_{X}$ and $j \circ m=f \circ \beta_{\Lambda X}$. Since $\Lambda_{X} \circ l \circ m=s_{M_{X}} \circ m=\Lambda_{X}=\Lambda_{X} \circ 1_{\Lambda X}$ and $\Lambda_{X}, l \circ m$ are coevring maps, $l \circ m=1_{\Lambda X}$. Hence $s_{M}^{-1}(X)$ and $\Lambda X$ are homeomorphic.

We recall that a space $X$ is called a weakly Lindelöf space if for any open cover $\mathcal{U}$, there is a countable subset $\mathcal{V}$ of $\mathcal{U}$ such that $\cup \mathcal{V}$ is dense in $X$ and that $X$ is called a countably locally weakly Lindelöf space if for any countable set $\left\{\mathcal{U}_{n} \mid n \in \mathbb{N}\right\}$ of open covers of $X$ and any $x \in X$, there is a neighborhood $G$ of $x$ in $X$ and for any $n \in \mathbb{N}$, there is a countable subset $\mathcal{V}_{n}$ of $\mathcal{U}_{n}$ such that $G \subseteq c l_{X}\left(\cup \mathcal{V}_{n}\right)$.

It was shown that for any countably locally weakly Lindelöf space $X, \Lambda_{\beta}^{-1}(X)$ is a basically disconnected space([8]). Using Lemma 2.4 and Theorem 3.2, we have the following corollary :

Corollary 3.3. Let $X$ be a countably locally weakly Lindelöf space. Then the set $\left\{M \mid M\right.$ is a $\sigma$-complete Boolean subalgebra of $\mathcal{R}(X)$ containg $Z(X)^{\#}$ and $s_{M}^{-1}(X)$ is basically disconnected $\}$, when partially ordered by inclusion, becomes a complete lattice. Moreover, $\sigma Z(X)^{\#}$ is the bottom element and $Z\left(\Lambda_{X}\right)^{\#}$ is the top element.

\section{REFERENCES}

1. J. Adámek, H. Herrilich \& G.E. Strecker: Abstract and concrete categories. John Wiley and Sons Inc. New York 1990.

2. A.M. Gleason: Projective topological spaces. 2 (1958), Illinois J. Math.

3. L. Gillman \& M. Jerison: Rings of continuous functions. Van Nostrand, Princeton, New York, 1960. 
4. M. Henriksen, J. Vermeer \& R.G. Woods: Quasi-F-covers of Tychonoff spaces. Trans. Amer. Math. Soc. 303 (1987), 779-804.

5. M. Henriksen, J.Vermeer \& R.G. Woods: Wallman covers of compact spaces. Dissertationes Mathematicae 283 (1989), 5-31.

6. S. Iliadis: Absolute of Hausdorff spaces. Sov. Math. Dokl. 4 (1963), 295-298.

7. C.I. Kim: Minimal covers and filter spaces. Topol. and its Appl. 72 (1996), 31-37.

8. __ : Minimal basically disconnected covers of product spaces. Commun. Korean. Math. Soc. 21 (2006), 347-353.

9. J. Porter \& R.G. Woods: Extensions and Absolutes of Hausdorff Spaces. Springer, Berlin, 1988.

10. J. Vermeer: The smallest basically disconnected preimage of a space. Topol. Appl. 17 (1984), 217-232.

${ }^{a}$ Department of Mathematics Education, ChonBuk National University, Jeonju 561756, RePublic OF KOREA

Email address: jyj@jbnu.ac.kr

${ }^{\text {b} D e p a r t m e n t ~ o f ~ M a t h e m a t i c s ~ E d u c a t i o n, ~ D a n k o o k ~ U n i v e r s i t y, ~ Y o n g i n ~ 448-701, ~ R e p u b-~}$ LIC OF KOREA

Email address: kci206@hanmail.net 